\title{
Pilot Trial of an Infant Screening Programme for Cystic Fibrosis: Measurement of Parotid Salivary Sodium at 4 months
}

\author{
DAVID LAWSON, PETER WESTCOMBE, and BARRY SAGGERS \\ From Queen Mary's Hospital for Children, Carshalton, Surrey; and \\ the Department of Health and Family Services, London Borough of Sutton
}

Cystic fibrosis (CF), transmitted by a single autosomal recessive gene, affects 1 in 2500 of the population. A biochemical abnormality affects the quality of mucus, and results in pancreatic duct obstruction, and progressive lung damage as a result of respiratory infection. Thirty years ago it is probable that almost all affected babies died of pneumonia in the first two years of life. Over the past generation the prognosis has improved substantially for children fortunate enough to be diagnosed early, and some of these are now reaching adult life with relatively little lung damage. There is, however, still substantial illness and mortality in childhood, and much of this could be avoided by earlier diagnosis. The lungs are structurally normal at birth, but have a built-in tendency to progressive lung damage; by the time respiratory problems lead to a diagnosis irreversible lung damage may have been done.

About one case a day is born in the United Kingdom, the average paediatrician sees about one new case a year, and the average family doctor will have one new case in his practice in his working life.

The natural history of CF suggests the desirability of making a diagnosis before it becomes clinically obvious. The requirements of a screening programme are that it should be harmless, reliable, accurate, cheap, and acceptable to the very large number of unaffected people who will be subjected to it. None of the existing methods of diagnosis satisfy the last two criteria. The most widely used diagnostic test is the measurement of sodium or chloride content of sweat, the flow of which has been stimulated by pilocarpine iontophoresis. Other exocrine glands are, however, similarly affected, and Johnston (1956) reported

Received May 12, 1969. that in CF the sodium content of parotid saliva was high.

The parotid salivary gland secretes continuously in the absence of specific stimulation, and its orifice is easily accessible. A micro-dual sodium sensitive electrode which could be used in the mouth was developed, and we proceeded to measure the saliva sodium concentration in a series of normals, heterozygous unaffected genetic carriers (i.e. parents), and cases of CF. The technique was described in detail by Saggers et al. (1967). Results indicated that consistent and reliable measurements could be made, and that there was a clear distinction between the sodium levels in normals and heterozygotes on the one hand, and cases of CF on the other.

Measurements were next made on a series of 50 babies in the first week of life, but these showed a very wide scatter of readings at high levels in the normal, presumably due to immaturity of control mechanisms. The same 50 babies tested at 6 weeks showed a reduced but still substantial scatter, and a lower mean, but still well above the levels found in normal people in later life (Lawson, Saggers, and Chapman, 1967). A third series of 50 babies was then tested in the clinics of the London Borough of Sutton at the age of 3-4 months, and it was found that by this age unstimulated parotid salivary sodium levels had reached maturity.

There was now a sufficient basis for a pilot trial of the technique, not only to confirm the findings on a larger scale, but to study the administrative problems of running such a programme. Screening was therefore offered to all 3-4-month-old babies in the London Borough of Sutton from February 1, 1968 , to January 30,1969 . This was a cooperative venture between a children's hospital and a Local Authority. The Local Authority's contri- 
bution in terms of clinic organization, staffing, correspondence, etc. was provided from within normal resources. The hospital's contribution was entirely covered by a research grant from the Cystic Fibrosis Research Trust.

The screening programme was approved in advance by the Health Committee of the London Borough of Sutton. This gave it some initial publicity in the local press which was probably helpful. The test was included in the description of the Local Authority's services given by Health Visitors at their first visits to all babies in the area: attendance for the test was encouraged, but it was made clear that it was experimental, and no general propaganda was undertaken. Special clinics were arranged for the purpose, and were held in normal clinic accommodation. No special equipment was required other than the electrometer and electrode which the technician brought from the hospital. The clinics were run by one nurse and one technician, and a clerk was responsible for sending appointments by post, these being accompanied by an explanatory letter. All the mothers who attended were co-operative, and there were no complaints about the procedure. Mothers who failed to attend the first appointment were sent a second, but not a third.

$54 \%$ of all mothers who were offered appointments attended. The figure would undoubtedly have been much higher if the propaganda and follow-up methods applicable to fully accepted procedures had been employed. If the test gains general acceptance we see no difficulty about including it in a Local Authority's routine services.

It is important that where babies are called for retesting, this should be done very quickly, and that results should be given to their parents without delay, otherwise a lot of unnecessary anxiety is created.

All the known sources of error in the method tend to result in falsely high readings. If parotid secretion is stimulated above its resting level by hunger, emotion, feeding, or vomiting, or by the placing of substances such as ascorbic acid in the mouth, the sodium reabsorbing mechanisms appear to become saturated, and the sodium concentration of saliva rises to higher levels. Whenever a high reading was obtained, the baby was retested on another occasion under ideal conditions, and a classical sweat test was done in parallel. In every case except one, the readings were well within the normal range. It was only found necessary to retest $2 \%$ of the babies.

One case was diagnosed at the age of 4 months. This baby has not as yet suffered any ill effects

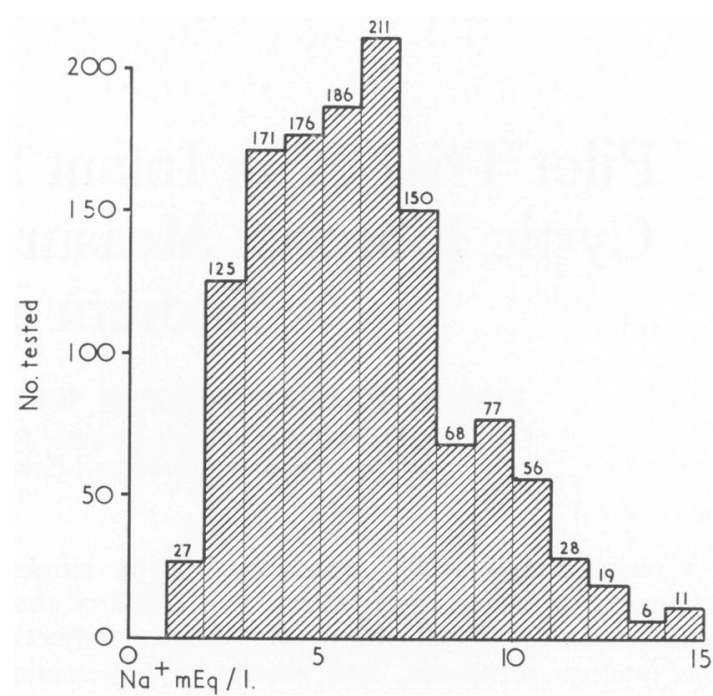

Fig. 1.-Distribution of parotid sodium levels in 1311 babies in whom the initial readings were below $15 \mathrm{mEq} / \mathrm{l}$.

from the disease, and is now under prophylactic care. When she was sent for, for confirmation of the diagnosis, her mother asked if she might also bring her 21-month-old child for screening as he appeared to have symptoms of the disease; and the diagnosis was confirmed in him also. He had had digestive and respiratory symptoms which had not been diagnosed.

Fig. 1 is a histogram showing the distribution of all initial readings of less than $15 \mathrm{mEq} / \mathrm{l}$. Fig. 2 is a histogram showing the initial and retest results

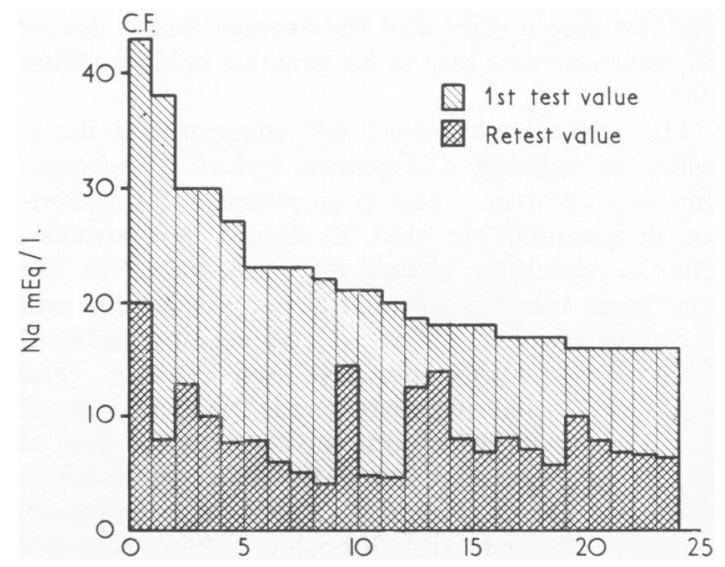

FIG. 2.-Initial and retest parotid sodium levels in 24 babies in whom the initial readings were above $15 \mathrm{mEq} / \mathrm{l}$. 
where initial readings were greater than $15 \mathrm{mEq} / 1$. (in this histogram each column refers to one child). Fig. 3 is a histogram showing the final distribution, including restest values where appropriate. It will be seen from Fig. 3 that the peak incidence of values is between 2 and $8 \mathrm{mEq} / 1$., and that $95 \%$ of values were below $10 \mathrm{mEq} / \mathrm{l}$.

There is not, however, a sharp cut off at 15 , and we propose in future to retest at a level of $13 \mathrm{mEq} / \mathrm{l}$. which will give a retest rate of just over $5 \%$.

Fig. 4 shows the parotid sodium levels found in 56 patients with CF (outside this series) who had been reliably diagnosed by other means. It will be seen that in 12 tested in the fourth month of life there were no readings below $17 \mathrm{mEq} / 1$; and that in 44 children over this age there were 3 readings between 12 and $15 \mathrm{mEq} / \mathrm{l}$. The ages of these 3 children were 4,10 , and 18 years.

The results of this pilot trial suggest that it would be well worth while running a large trial covering an area with 10,000 births per annum, when it could be expected that 4 cases would be diagnosed per annum. Such a trial is planned in this area.

There is no point in making an early diagnosis unless early action follows; and any such screening programme should be organically linked with a CF unit in the hospital service where any diagnostic problems which arise can be immediately sorted out, and where in the cases diagnosed a fully

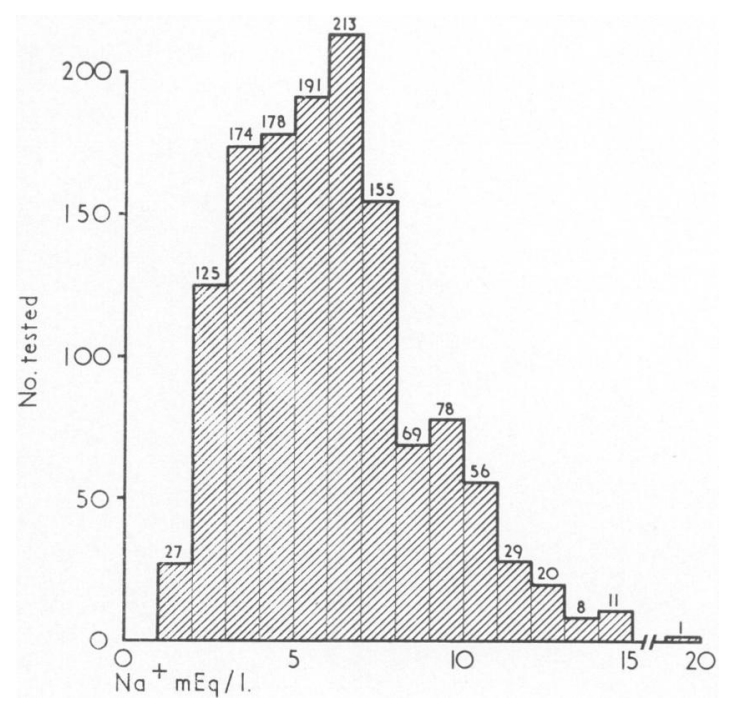

FIG. 3.-Final distribution of parotid sodium levels in 1335 babies (including retest values).

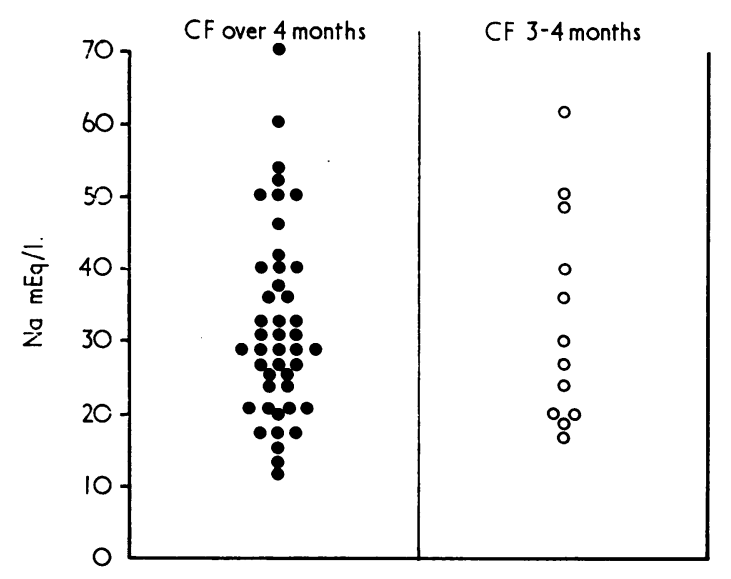

FIg. 4.-Parotid salivary sodium levels in 56 known cases of $C F$ (separate series).

prophylactic programme can be started at once, including physiotherapy and respiratory tuition, pancreatin replacement therapy and dietary guidance, and prophylactic antistaphylococcal therapy.

\section{Appendix on Cost}

It is difficult to cost precisely an operation which makes marginal use of the normal resources of both a hospital and a Local Authority. We made accurate records over a series of 1000 examinations in 9 months, and the results are expressed in shillings per child screened.

Local Authority. Nursing time in special clinics, secretarial time, and postage were included. Overheads of clinic buildings, and normal Health Visitor visits were excluded. The cost to the Local Authority per examination was $2 \mathrm{~s}$. $6 \mathrm{~d}$. $(£ 0 \cdot 125)$.

Hospital. Junior technician time at the clinics and travelling, motor car transport costs, and laboratory time spent on retests were included. Supervision and training of the technician, and minor use of secretarial time were excluded: as were the capital costs of the electrometer and electrode. The electrometer is standard laboratory equipment. Electrodes cost about $£ 10$ each and last a long time. The cost to the hospital per examination was 1s. 10d. ( $£ 0.092)$.

The total calculated cost per screening was therefore 4s. $4 d$. ( $£ 0 \cdot 217)$, of which $57 \%$ was borne by the local authority and $43 \%$ by the hospital.

Assuming an incidence of CF of 1 in 2500 births, the cost per case diagnosed would be about $£ 540$. The case for screening is not, of course, a financial one; but even in financial terms this appears cheap compared with the cost of frequent preventable in-patient treatment at over $£ 50$ per week. 
In terms of technician time, it appears that 12,000 examinations per annum would require the equivalent of one whole-time technician. In practice it would be desirable to split such a load of repetitive work between several technicians.

\section{Summary}

In a pilot trial in which 1335 4-month-old babies were screened for cystic fibrosis by measuring the sodium content of unstimulated parotid saliva with a sodium sensitive electrode, the cost was less than $5 \mathrm{~s}$. per child screened, and one case of cystic fibrosis was diagnosed.

The screening technique proved acceptable and reliable, and should be applied in larger scale trials.
We wish to express our thanks to the Health Committee of the London Borough of Sutton for their cooperation; to the Cystic Fibrosis Research Trust who covered all expenditure on the hospital side of the project; and to Junior Technicians, James Kendra and Beverley Goldsworthy, who carried out the majority of the tests.

\section{REFERENCES}

Johnston, W. H. (1956). Salivary excretions in fibrocystic disease of the pancreas. Arch. Dis. Childh., 31, 477.

Lawson, D., Saggers, B. A., and Chapman, M. J. (1967). Screening for cystic fibrosis by measurement of unstimulated parotid saliva sodium levels. ibid., 42, 689.

Saggers, B. A., Lawson, D., Stern, J., and Edgson, A. C. (1967). Rapid method for the detection of cystic fibrosis of the pancreas in children. ibid., 42, 187.

Correspondence to Dr. David Lawson, Queen Mary's Hospital for Children, Carshalton, Surrey. 